\title{
The effect of CYP2D6 * 10 polymorphism on adjuvant tamoxifen in Asian breast cancer patients: a meta-analysis
}

\author{
Junjun $L u^{1, *}$ \\ $\mathrm{He} \mathrm{Li}^{2}$,* \\ Peng Guo' \\ Rui Shen ${ }^{3}$ \\ Yingbin Luo' \\ Qiao $\mathrm{Ge}^{4}$ \\ Wenfei Shi' \\ Yan $\mathrm{Li}^{\prime}$ \\ Weikang Zhu' \\ 'Department of Tumor, Shanghai \\ Municipal Hospital of Traditional \\ Chinese Medicine, ${ }^{2}$ Department \\ of Traditional Chinese Medicine, \\ Shanghai Jiao Tong University Medical \\ School Affiliated Ruijin Hospital, \\ ${ }^{3}$ Department of Function, Shanghai \\ Municipal Hospital of Traditional \\ Chinese Medicine, Shanghai, \\ ${ }^{4}$ Department of Tumor, Ninghai \\ Hospital of Traditional Chinese \\ Medicine, Ningbo, Zhejiang, China \\ *These authors contributed equally \\ to this work
}

\author{
This article was published in the following Dove Press journal: \\ OncoTargets and Therapy \\ 13 November 2017 \\ Number of times this article has been viewed
}

Objective: To evaluate the effect of $C Y P 2 D 6 * 10$ polymorphism $(\mathrm{C} 100 \mathrm{C}>\mathrm{T}$, rs 1065852) on clinical outcomes of female Asian breast cancer patients with tamoxifen adjuvant treatment.

Methods: Meta-analysis of retrospective cohort studies published in July 2017 was performed. Fifteen studies with 1,794 Asian breast cancer patients were included, using strict eligibility requirements. Associations of disease-free survival (DFS), overall survival (OS) and recurrence rate after tamoxifen intake, with $C Y P 2 D 6 * 10$ polymorphism were investigated through random effects models.

Results: $C Y P 2 D 6 * 10$ polymorphism was found to have effect on DFS and recurrence rate in various comparison models, but not on overall survival in the female Asian breast cancer patients.

Conclusion: In conclusion, our meta-analysis suggests that significant association of $* 10 / * 10$ (TT) genotype with poorer DFS and recurrence exists in female Asian breast cancer patients with tamoxifen $20 \mathrm{mg}$ /day adjuvant treatment. In the future, large and well-designed studies are required to illustrate the interactions of CYP2D6 genetic variants, including * 10 polymorphism and tamoxifen response on female breast cancer patients.

Keywords: $C Y P 2 D 6 * 10$, polymorphism, breast cancer, Asia, tamoxifen adjuvant treatment

\section{Introduction}

Tamoxifen has been widely used for $>30$ years as adjuvant treatment for women operated for estrogen receptor-positive breast cancer. It is reported that postoperative 5 -year tamoxifen therapy effectively reduces the risk of breast cancer recurrence by $39 \%{ }^{1}$ and breast cancer mortality. ${ }^{1}$

The clinical efficacy of tamoxifen adjuvant treatment varies among individuals. Tamoxifen itself has weak affinity for the estrogen receptor and undergoes considerable first-pass oxidative metabolism to more potent metabolites, such as 4-hydroxytamoxifen and 4-hydroxy-N-desmethyl tamoxifen (endoxifen), which is the main active tamoxifen, and has up to a 33-fold higher affinity for the estrogen receptor than tamoxifen itself. $^{2}$ The rate-limiting step in tamoxifen metabolism is mediated primarily by the highly polymorphic CYP2D6. ${ }^{3}$

Several studies have reported that $C Y P 2 D 6$ genetic mutations leading to the absence of functional enzyme are related to the decrease in 4-hydroxytamoxifen and endoxifen levels. ${ }^{4}$ However, it is still highly controversial on the clinical association of CYP2D6 genetic variants as predictors of tamoxifen efficacy at the standard dose of $20 \mathrm{mg} /$ day. Nonfunctional allele variants of CYP2D6 gene were reported to be associated with increased risk of breast cancer relapse in female breast cancer patients ${ }^{4-8}$ and increased 
risk of breast cancer occurrence in unaffected women. ${ }^{9}$ Meanwhile, other studies do not support such an association. ${ }^{10-13}$ The causes of these conflicting results might be clarified by some newly emerged evidence, ${ }^{14,15}$ such as inclusion criteria for patients, source of deoxyribonucleic acid and genotyping method, coverage of genetic mutations and polymorphisms, as well as serological concentrations of endoxifen.

The classical CYP2D6-predicted phenotype is commonly divided into 4 levels of enzyme activity: poor metabolizers (PMs), intermediate metabolizers (IMs), extensive metabolizers and ultrarapid metabolizers. ${ }^{16}$ To date, more than 100 allelic variants have been described for CYP2D6 gene. ${ }^{17}$ CYP2D6 * 10 is a major variant and produces an unstable CYP2D6 enzyme, with an allele frequency of $\sim 50 \%$ in Asians ${ }^{18}$ but only $2 \%$ in Caucasians. ${ }^{19}$ Mutation of CYP $2 D 6 * 10$ generates a $188 \mathrm{C}$ to $\mathrm{T}$ transition in exon 1 , leading to a Proline 34 to Serine amino acid substitution and resulting in an unstable enzyme with lower activity and metabolic conversion rate. ${ }^{20}$

It would be of great interest to investigate whether CYP2D6 * 10 genotype affects the efficacy of tamoxifen in female Asian breast cancer patients. To test this hypothesis, we investigated whether the CYP2D $6 * 10$ genotype is associated with the clinical outcome in a particular population subgroup of female Asian breast cancer patients by metaanalysis.

\section{Methods}

\section{Literature and search strategy}

The PubMed, MEDLINE, Cochrane, Embase, Wan Fang and Chinese National Knowledge Infrastructure database searches were conducted for all the pertinent trials. The search terms were: "CYP2D6" and "polymorphism" and "tamoxifen" and "breast cancer". Manual scrutiny for eligible articles was conducted according to the bibliography of the original and review reports. The literature search was updated in July 2017.

\section{Study selection}

Retrieved studies were deemed eligible provided that they met all the following criteria: 1) studies on human beings; 2 ) in a cohort design or randomized controlled trial design; 3 ) investigated the effect of $C Y P 2 D 6 * 10$ polymorphism with outcome of breast cancer patients taking tamoxifen; 4) in Asian population; 5) treated with $20 \mathrm{mg}$ /day adjuvant tamoxifen for 2-5 years; 6) detailed outcomes could be obtained or calculated on the outcome of disease-free survival (DFS), overall survival (OS) or recurrence rate; 7) the number of women with breast cancer was $>30 ; 8$ ) and received more than 4 points in the Newcastle-Ottawa Scale (NOS), which was considered to be of high quality.

\section{Data extraction}

Three reviewers screened titles and abstracts for relevance to the topic independently (Junjun Lu, He Li and Peng Guo). All potential publications were independently evaluated in full text. The following information was sought for reaching consensus on all of the items: the first author's name, year of publication, design of trial, comparison, number of patients, and the endpoints of clinical prognosis. Any disagreements were resolved by the fourth investigator (Zhu Weikang).

\section{Statistical analysis}

Five genic comparison models (TT vs CC, CT vs CC, TT vs $\mathrm{CT} / \mathrm{CC}$, TT/CT vs CC, and TT vs CT) for 3 endpoints (DFS, OS and reference) were analyzed. A $\chi^{2}$-test based on the Q test and $I^{2}$ metric were used to quantify the heterogeneity. ${ }^{21}$ When $I^{2}>50 \%$ and $p<0.1$, the between-study heterogeneity was considered to be significant and the random-effects model was used. Otherwise, for homogeneous studies, the fixed effects model was used. The mean hazard ratios (HRs) and 95\% CIs were obtained from the original article by using survival analysis. ${ }^{22,23}$ If a publication did not provide HR values, Engauge Digitizer version 4.1 was used to obtain the HR values and 95\% CIs. ${ }^{23}$ The pooled HR was initially demonstrated using forest plots graphically. Begg's Funnel plot and Egger's test were conducted to evaluate the potential publication bias. ${ }^{24}$ Sensitivity analysis was performed to assess the stability of our outcomes by omitting each study sequentially. Hardy-Weinberg equilibrium was examined with goodness-of-fit $\chi^{2}$-test. Two-sided $p<0.05$ was considered to be statistically significant. STATA version 12.0 (StataCorp LLC, College Station, TX, USA) was used for all the data analyses and graphs.

\section{Results}

\section{Characteristics of studies included in this meta-analysis}

Initial search yielded a total of 221 potentially relevant citations. Finally, 15 studies ${ }^{7,25-38}$ were included in our metaanalysis to meet the inclusion criteria (Figure 1). Publication years ranged from January 2008 to July 2017; the number of breast cancer patients varied from 39 to 296 (Table 1). As a result, data for our meta-analysis were available from 15 studies with a total of 1,794 Asian breast cancer patients. The eligible studies were assessed by the NOS. Each of the 


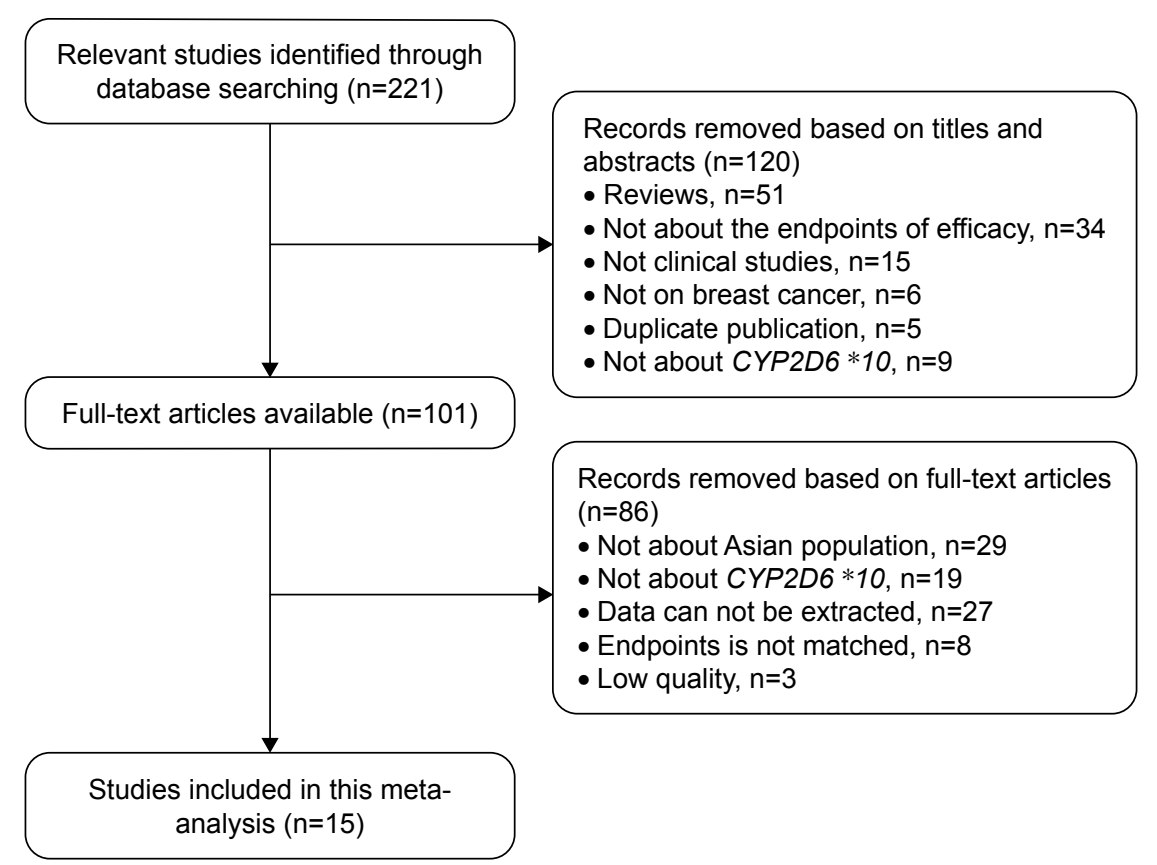

Figure I Study flow diagram for the process of selecting the final I5 studies.

studies scored $>4$, which suggested that all the research was of high quality (Table 1).

\section{Meta-analysis results}

The pooled effect of CYP2D6*10 genotype on the DFS of breast cancer patients treated with tamoxifen is shown in Figure 2 and Table 2. CYP2D6 *10 polymorphism (C 100C $>\mathrm{T}$, rs1065852) significantly influences DFS in various comparison models (TT vs $\mathrm{CC}$ : $\mathrm{HR}=1.79$, 95\% CI $=1.14-2.80, p=0.011$; CT vs CC: $\mathrm{HR}=2.02$,
95\% CI $=1.04-3.19, p=0.037$; TT vs $\mathrm{CC}: \mathrm{HR}=1.79$, 95\% CI $=1.14-2.80, p=0.011$; TT vs CT: $\mathrm{HR}=2.03$, $95 \% \mathrm{CI}=1.41-2.93, p<0.001$; TT vs CT/CC: HR $=2.19$, $95 \% \mathrm{CI}=1.07-4.50, p=0.033)$ except the dominant model (TT/CT vs CC: $\mathrm{HR}=1.80,95 \% \mathrm{CI}=0.81-4.00, p=0.147$ ).

\section{Heterogeneity}

There was no significant between-study heterogeneity for the endpoint of DFS in the comparison model of TT vs CC $\left(I^{2}=44.5 \%, p=0.114\right)$, CT vs CC $\left(I^{2}=36.3 \%, p=0.194\right)$ and

Table I Characteristics and quality assessment of the included studies

\begin{tabular}{|c|c|c|c|c|c|c|c|c|}
\hline Study ID & Country & $\begin{array}{l}\text { Sample } \\
\text { size }\end{array}$ & $\begin{array}{l}\text { Menopause status } \\
\text { (pre + post) }\end{array}$ & $\begin{array}{l}\text { Follow-up } \\
\text { (months) }^{\mathrm{a}}\end{array}$ & Endpoints & $\begin{array}{l}\text { Genotyping } \\
\text { method }\end{array}$ & HWE & NOS \\
\hline Xu et $\mathrm{al}^{35}$ & China & 152 & - & $63(4-122)$ & DFS & RT-PCR & $Y$ & 6 \\
\hline Kiyotani et $\mathrm{al}^{26}$ & Japan & 67 & $35+32$ & $96(19.2-259.2)$ & RFS, recurrence & TaqMan & $Y$ & 7 \\
\hline Sirachainan et $\mathrm{al}^{29}$ & Thailand & 39 & $3 I+8$ & $52.3-97.4$ & DFS & Pyrosequencing & $Y$ & 6 \\
\hline Sukasem et $\mathrm{al}^{30}$ & Thailand & 48 & $30+18$ & 67.2 & DFS & Microarray & $Y$ & 7 \\
\hline Teh et $\mathrm{al}^{7}$ & Mixed* & 95 & $33+62$ & - & Recurrence & TaqMan & $Y$ & 7 \\
\hline Chamnanphon et $\mathrm{al}^{25}$ & Thailand & 57 & $38+19$ & $48(2-172)$ & DFS, recurrence & Microarray & $Y$ & 6 \\
\hline Tian et $\mathrm{a}^{31}$ & China & 200 & - & $31.5(9-58)$ & DFS & TaqMan & $Y$ & 7 \\
\hline Wei and $X u^{33}$ & China & 257 & - & - & DFS, OS & RT-PCR & $Y$ & 5 \\
\hline Xiao et $\mathrm{al}^{34}$ & China & 87 & $0+87$ & $35(12-43)$ & DFS, recurrence & TaqMan & $Y$ & 6 \\
\hline Wang et $\mathrm{al}^{32}$ & China & $17 \mid$ & - & 60 & OS & RT-PCR & $Y$ & 5 \\
\hline Zhang et $\mathrm{al}^{38}$ & China & 296 & $174+122$ & - & OS & Sequencing & $\#$ & 6 \\
\hline Lei et $\mathrm{al}^{27}$ & China & 72 & $65+7$ & $85(20-144)$ & DFS, OS & Pyrosequencing & $Y$ & 6 \\
\hline Sensorn et $\mathrm{al}^{28}$ & Thailand & 73 & $47+26$ & $87.6(60-17 \mid .6)$ & DFS & RT-PCR & $Y$ & 7 \\
\hline Yu et $\mathrm{al}^{36}$ & China & 106 & - & - & DFS & RT-PCR & $Y$ & 5 \\
\hline Zeng et $\mathrm{al}^{37}$ & China & 76 & - & $30.5(5-40)$ & Recurrence, OS & RT-PCR & $Y$ & 5 \\
\hline
\end{tabular}

Notes: *Mixed includes patients in Malaysia, China, and India. ${ }^{H} \mathrm{HWE}$ of Zhang et al ${ }^{38}$ was unknown and could not be estimated. aData presented as median (range). Abbreviations: DFS, disease-free survival; HWE, Hardy-Weinberg equilibrium; NOS, Newcastle-Ottawa scale; OS, overall survival; RFS, relapse-free survival; RT-PCR, real time-polymerase chain reaction. 


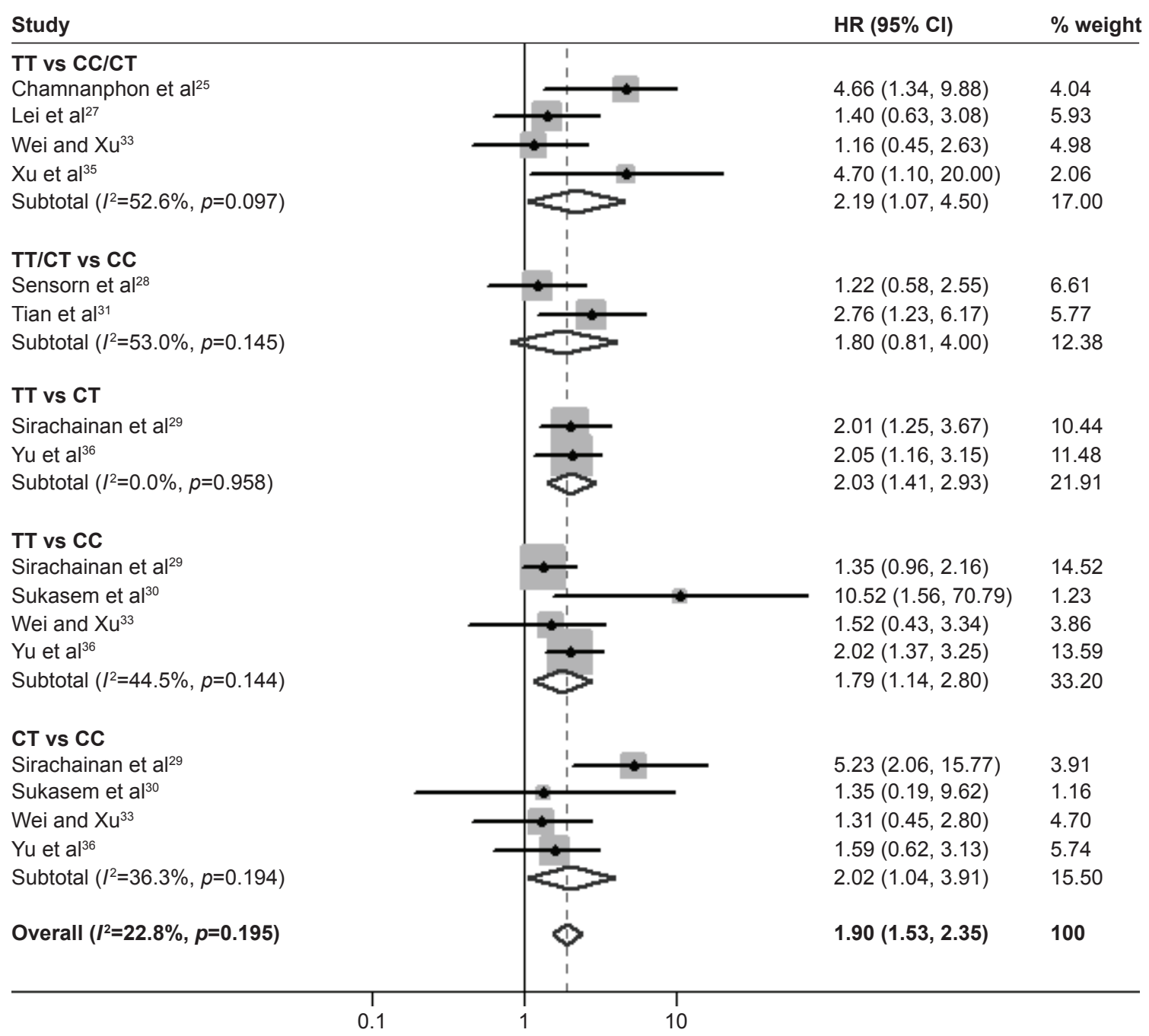

Figure 2 Forest plot to estimate the effect of CYP2D6 *10 polymorphism on the DFS of breast cancer patients taking tamoxifen.

Note: Weights are from random effects analysis.

Abbreviations: DFS, disease-free survival; HR, hazard ratio.

TT vs CT $\left(I^{2}=0 \%, p=0.958\right)$. Meanwhile, for the dominant model and recessive model, heterogeneity was detected to be significant. Thus, random-effects estimates would be more appropriate for data analysis. Similarly, the random-effect model was conducted for the comparison of TT vs CC for OS and $\mathrm{T}$ allele vs $\mathrm{C}$ allele for recurrence (Tables 3 and 4).

\section{Publication bias and sensitivity analysis}

The publication bias of literature was assessed with both funnel plots and Egger's test. As shown in Tables 2-4 and Figure 4, our results did not reveal any obvious asymmetry in the funnel plots. Moreover, the Egger's test, which was used to provide statistical evidence of publication bias, suggested

Table 2 Results of the effect of CYP2D6 *10 polymorphism on the DFS of breast cancer patients taking tamoxifen

\begin{tabular}{|c|c|c|c|c|c|c|c|c|}
\hline \multirow[t]{2}{*}{ Comparison } & \multirow[t]{2}{*}{ Studies } & \multicolumn{3}{|l|}{ Overall effect } & \multicolumn{2}{|c|}{ Heterogeneity } & \multicolumn{2}{|c|}{ Publication bias } \\
\hline & & HR (95\% CI) & $\mathbf{Z}$ & $p$-value & $I^{2}, \%$ & $p$-value & Begg's test & Egger's test \\
\hline TT vs CC & 4 & I.79 (I.I4-2.80) & 2.54 & 0.011 & 44.50 & 0.144 & I & 0.91 \\
\hline CT vs CC & 4 & 2.02 (1.04-3.91) & 2.09 & 0.037 & 36.30 & 0.194 & 0.734 & 0.351 \\
\hline TT vs CT/CC & 4 & $2.19(1.07-4.50)$ & 2.13 & 0.033 & 52.60 & 0.097 & 0.308 & 0.012 \\
\hline TT/CT vs CC & 2 & $\mathrm{I} .80(0.8 \mathrm{I}-4.00)$ & 1.45 & 0.147 & 53.00 & 0.145 & I & - \\
\hline TT vs CT & 2 & $2.03(1.4 I-2.93)$ & 3.79 & 0 & 0.00 & 0.958 & I & - \\
\hline
\end{tabular}

Notes: Different from DFS, the pooled results of the endpoint of OS showed that there was no relationship between CYP2D6 $* 10$ polymorphism and OS of female breast cancer patients with adjuvant tamoxifen. As shown in Table 3, all the genetic models did not support any evidence of association (TT vs CC: $\mathrm{HR}=\mathrm{I} .05,95 \% \mathrm{Cl}=0.35-3.1 \mathrm{I}$, $p=0.934$; $C T$ vs $C C: H R=1.29,95 \% \mathrm{Cl}=0.83-2.00, p=0.225$; TT vs $C T / C C: H R=0.96,95 \% \mathrm{Cl}=0.62-1.48, p=0.858 ;$ TT/CT vs CC: $\mathrm{HR}=1.57,95 \% \mathrm{Cl}=0.89-2.77, p=0.119)$. For the additive comparison model of TT vs CT, which was reported by only one study, the result was negative as well.

Abbreviations: DFS, disease-free survival; HR, hazard ratio; OS, overall survival. 
Table 3 Results of the effect of CYP2D6 *10 polymorphism on the OS of female breast cancer patients taking tamoxifen

\begin{tabular}{|c|c|c|c|c|c|c|c|c|}
\hline \multirow[t]{2}{*}{ Comparison } & \multirow[t]{2}{*}{ Studies } & \multicolumn{3}{|l|}{ Overall effect } & \multicolumn{2}{|c|}{ Heterogeneity } & \multicolumn{2}{|c|}{ Publication bias } \\
\hline & & HR $(95 \% \mathrm{Cl})$ & $\mathbf{Z}$ & $p$-value & $I^{2}, \%$ & $p$-value & Begg's test & Egger's test \\
\hline TT vs CC & 3 & $1.05(0.35-3.11)$ & 0.08 & 0.934 & 73.10 & 0.024 & 1.00 & 0.320 \\
\hline CT vs CC & 3 & $1.29(0.83-2.00)$ & 1.14 & 0.255 & 0.00 & 0.844 & 1.00 & 0.751 \\
\hline TT vs CT/CC & 2 & $0.96(0.62-1.48)$ & 0.18 & 0.858 & 0.00 & 0.578 & 1.00 & - \\
\hline TT/CT vs CC & 2 & I.57 (0.89-2.77) & 1.56 & 0.119 & 0.00 & 0.468 & 1.00 & - \\
\hline TT vs CT & I & $1.36(0.58-3.18)$ & 0.71 & 0.478 & - & - & - & - \\
\hline
\end{tabular}

Notes: The results of the endpoint of recurrence are shown in Figure 3 and Table 4. CYP2D6 $* 10$ polymorphism was found to be associated with the increased risk of relapse of breast cancer in different genetic models (TT vs CC: OR $=4.07,95 \% \mathrm{Cl}=1.88-8.80, p<0.00 \mathrm{I}$; TT vs CT/CC: OR $=3.69, \mathrm{OR}=2$. I3-6.4I, $p<0.00 \mathrm{I}$; TT/CT vs CC: 2.52 , $95 \% \mathrm{Cl}=1.24-5.13, p=0.01 \mathrm{I} ; \mathrm{T}$ allele vs $\mathrm{C}$ allele: $2.99,95 \% \mathrm{Cl}=1.46-6.1 \mathrm{I}, \mathrm{p}=0.029)$. However, in the heterozygous model, heterozygous variant of $\mathrm{CYP} 2 \mathrm{D} 6 * 10$ gene did not increase the risk of relapse (CT vs $C C$ : $O R=1.39,95 \% \mathrm{Cl}=0.62-3.13, p=0.427$ ).

Abbreviations: HR, hazard ratio; OS, overall survival.

that no evidence of publication bias existed in the overall analysis ( $p>0.05$ for all the comparisons). Sensitivity analyses showed that omitting each individual study from all the analyses did not affect the pooled odds ratios significantly, and no substantial change was detected, indicating that our results were statistically robust (Figure 5).

\section{Discussion}

The CYP2D6 gene has earned special attention because an increasing number of studies have revealed that polymorphisms of the CYP2D6 gene were associated with the outcomes following tamoxifen treatment for breast cancer. ${ }^{39}$ However, the results across studies have been equivocal. ${ }^{40,41}$ Previous meta-analyses were performed by Jung and Lim, ${ }^{17}$ Province et $\mathrm{al}^{42}$ and Johansson et al. ${ }^{43}$ These meta-analyses were designed to evaluate the effect of CYP2D6 metabolizer types on outcome of breast cancer patients with tamoxifen treatment, for all the ethnicities. Different from these previous studies, our investigation aimed to assess the effect in a certain population and for a certain polymorphism, and was conducted in Asian breast cancer patients for CYP2D6 *10 polymorphism. Therefore, to our knowledge, it would be the first meta-analysis to report the unique association between CYP2D6 *10 and efficacy of tamoxifen in Asian breast cancer patients.

Ethnic difference in genetic background is one of the most important biological factors that might influence the patients' clinical response and efficacy. ${ }^{44} C Y P 2 D 6 * 10$ variant is commonly reported in Asian population, but not in other ethnicities. ${ }^{19}$ The genetic discrepancies might be one of the reasons for the dispute on effect of CYP2D6 variants on tamoxifen response. Besides, the coverage of CYP2D6 mutations in the different studies might also play a role in the dispute. ${ }^{20}$ Most research studies reported CYP2D6 metabolizer types with their effect of tamoxifen efficacy, however, CYP2D6-predicted phenotype classified system was too variable to reach a consensus. Therefore, it is of great clinical significance to conduct this meta-analysis to reveal the effect of $C Y P 2 D 6 * 10$ polymorphism on the tamoxifen efficacy in Asian patients. It might provide a new insight on a range of outcomes, investigating a potential gene dose-response relationship, and an assessment of data from trials that investigated effect modification of tamoxifen by CYP2D6 genotype status.

In the process of study inclusion, we selected several indicators, including DFS, OS, and recurrence rate as the endpoints of our meta-analysis. These indicators were commonly reported by various studies and were suitable to be pooled. In fact, 2 of the studies included ${ }^{26,45}$ reported the outcome of relapse-free survival (RFS). However, we failed to pool the results of RFS because these studies were with a different genetic comparison model. Similarly, data of disease-specific survival, time to progression, recurrence-free time were also reported by some studies but not available to be pooled.

Table 4 Results of the effect of CYP2D6 $* 10$ polymorphism on the recurrence of breast cancer in patients taking tamoxifen

\begin{tabular}{|c|c|c|c|c|c|c|c|}
\hline \multirow[t]{2}{*}{ Comparison } & \multicolumn{3}{|l|}{ Overall effect } & \multicolumn{2}{|c|}{ Heterogeneity } & \multicolumn{2}{|c|}{ Publication bias } \\
\hline & OR (95\% Cl) & $\mathbf{Z}$ & $p$-value & $I^{2}, \%$ & $p$-value & Begg's test & Egger's test \\
\hline TT vs CC & $4.07(1.88-8.80)$ & 3.57 & 0 & 40.20 & 0.154 & 0.22 & 0.11 \\
\hline CT vs CC & $1.39(0.62-3.13)$ & 0.79 & 0.427 & 0.00 & 0.725 & 0.462 & 0.331 \\
\hline TT vs CT/CC & $3.69(2 . \mid 3-6.4 I)$ & 4.65 & 0 & 40.90 & 0.148 & 0.806 & 0.51 \\
\hline $\mathrm{TT} / \mathrm{CT}$ vs CC & $2.52(1.24-5.13)$ & 2.55 & 0.011 & 14.10 & 0.325 & 0.22 & 0.073 \\
\hline T vs $C^{*}$ & $2.99(1.46-6.11)$ & 3 & 0.003 & 63.10 & 0.029 & 0.462 & 0.141 \\
\hline
\end{tabular}

Note: *Random effects model was used for the comparison of allele genetic model as heterogeneity is significant.

Abbreviation: OR, odds ratio. 
A

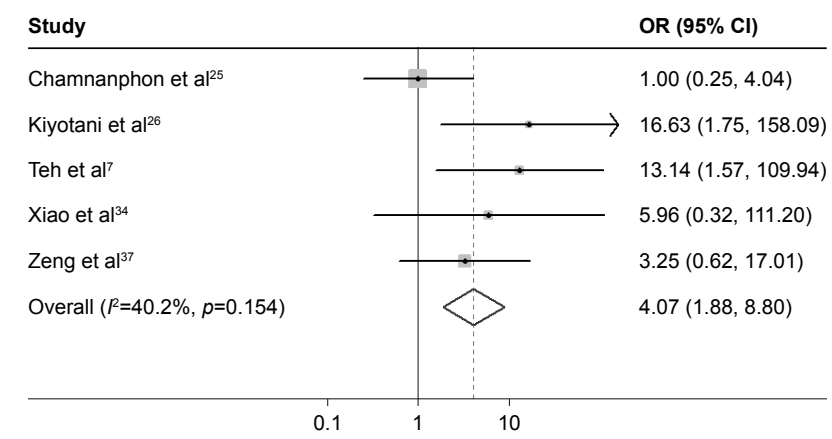

C

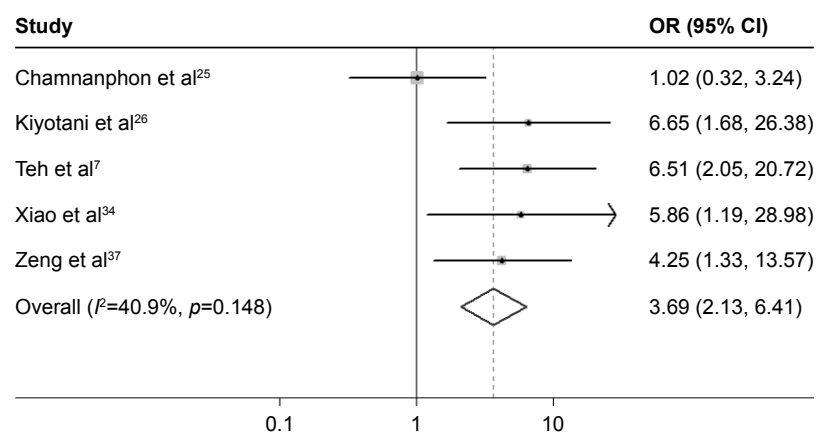

B

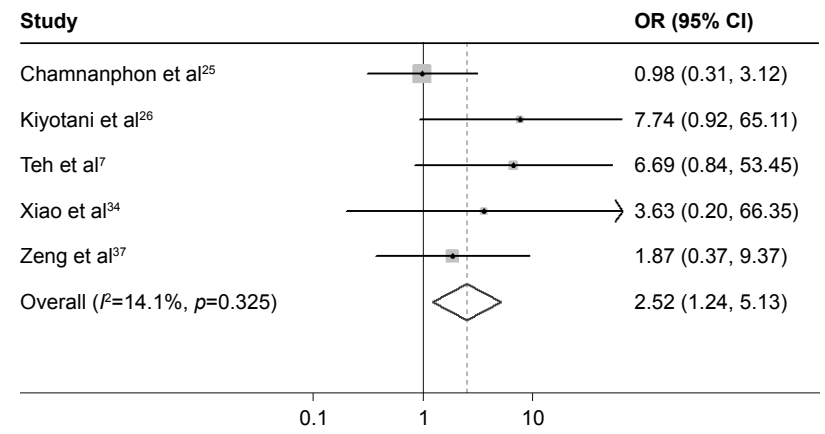

D

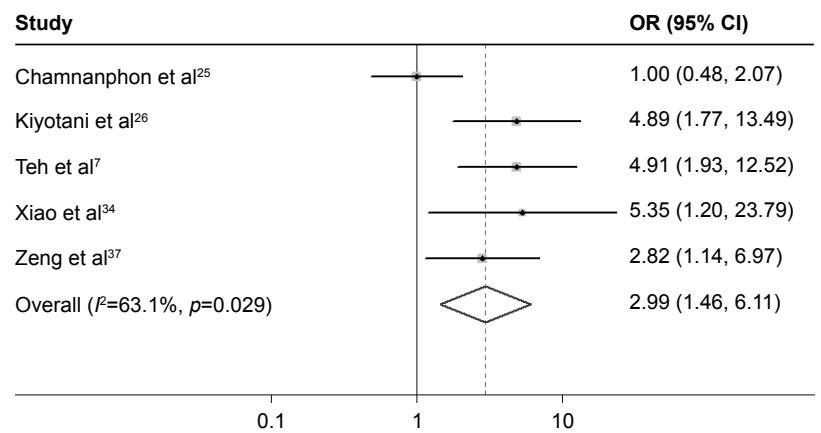

Figure 3 Forest plot to estimate the effect of CYP2D6 *10 polymorphism on the recurrence of breast cancer in patients taking tamoxifen in the comparison of (A) TT vs CC; (B) CT/TT vs CC; (C) TT vs CT/TT and (D) T vs C allele.

Note: Weights are from random effects analysis.

Abbreviations: $\mathrm{Cl}$, confidence interval; OR, odds ratio.

Tamoxifen will still be commonly used in both premenopausal and postmenopausal women in the foreseeable future. Therefore, analysis of CYP2D6 *10 genotype may be useful in identifying patients who are more likely to benefit from tamoxifen treatment. In fact, men with breast cancer are also an important subgroup who are treated in adjuvant setting preferentially with tamoxifen. CYP2D $6 * 4$ polymorphism was reported to be associated with a probability of recurrence in male breast cancer. ${ }^{46}$ Therefore, analysis of CYP2D6 $* 10$ variant in male breast

\section{A Begg's funnel plot with pseudo $95 \%$ confidence limits}

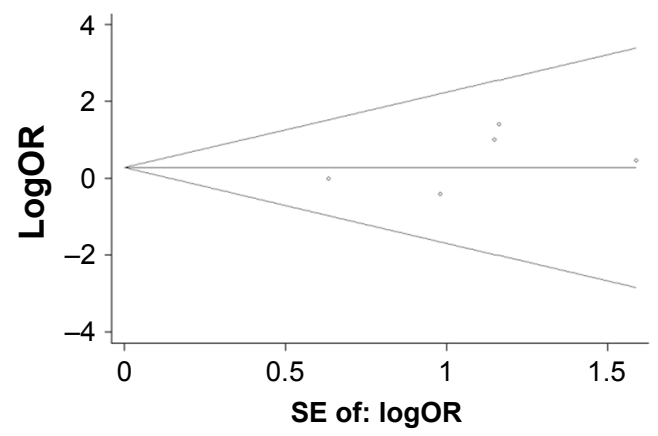

cancer would also be of great clinical significance. Our meta-analysis indicated the relationship of CYP $2 D 6 * 10$ polymorphism with tamoxifen response existed both on the endpoints of DFS and recurrence rate in female Asian breast cancer patients, with tamoxifen $20 \mathrm{mg}$ /day adjuvant therapy. It is consistent in direction with the hypothesis that reduced tamoxifen metabolism is associated with poorer outcome. ${ }^{5,11,30}$ In the genetic models of TT/CT vs CC in DFS and CT vs CC in recurrence, pooled results were negative for revealing the association of CYP2D6 $* 10$ and

\section{B Begg's funnel plot with pseudo $95 \%$ confidence limits}

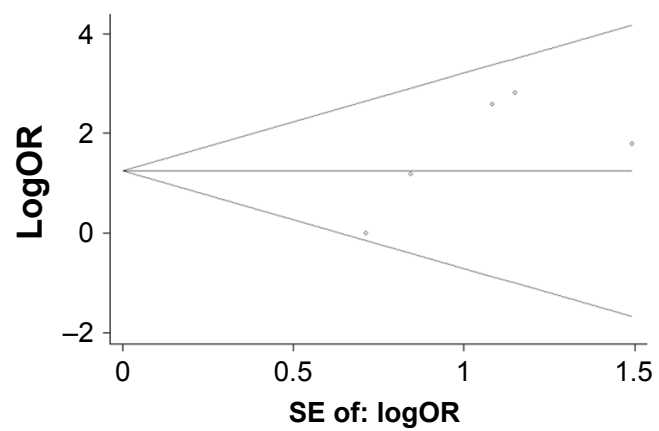

Figure 4 Begg's funnel plots to examine publication bias for reported comparisons of CYP2D6 *I0 polymorphism with recurrence in the comparison of (A) CT vs CC and (B) TT vs CC in Asian breast cancer patients treated with tamoxifen.

Abbreviations: OR, odds ratio; SE, standard error. 


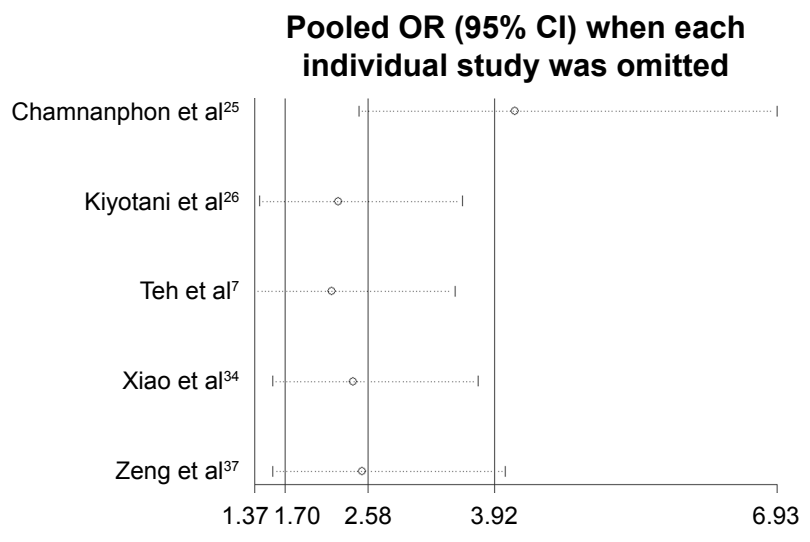

Figure $\mathbf{5}$ Sensitivity analysis of the comparison of allele genetic model ( $T$ vs $C$ allele) in the recurrence analysis.

tamoxifen efficacy. The former might be invalid, because this comparison contains only 2 studies, with significant heterogeneity $\left(I^{2}=53 \%, p=0.145\right)$, and is in contradiction to the other comparison, especially in TT vs CC and CT vs CC. For the $\mathrm{CT}$ vs $\mathrm{CC}$ comparison in recurrence, the results are without any between-study heterogeneity, and considered to be reliable. Our results suggests the decrease in enzyme activity caused by heterozygous mutation of CYP2D6 * 10 does not translate into a significant poorer clinical outcome. Meanwhile, $C Y P 2 D 6 * 10$ polymorphism was not associated with OS after pooled analysis, which was not consistent with the hypothesis. Given that OS is defined as the time from randomization to all-cause mortality, which is different from DFS, it might include in some death records that is irrelevant to cancer. Besides, for the endpoint of OS, 3 studies and 2 studies were pooled in different comparisons, which is relatively few and may have underpowered the results.

The heterogeneity between the studies was very low in our analysis. This suggested that the results from these studies were suitable to be pooled. The sensitivity analysis indicated that studies contributing to the heterogeneity did not significantly alter the pooled results. This suggested that our results were statistically robust. Taken together, our results might provide insights into the individualized therapy of breast cancer in Asian women. In patients with *10/*10 (TT) genotype, CYP2D6 enzyme activity and metabolic conversion rate was low. It is reported that increasing the regular tamoxifen dose could significantly increase endoxifen exposure in patients with CYP2D6 PMs or IMs. ${ }^{47}$ Therefore, female breast cancer patients with $* 10$ variant should increase their tamoxifen dose to $>20 \mathrm{mg} /$ day. However, the optimal dose of tamoxifen for $* 10$ carriers needs to be explored.

Several limitations in our meta-analysis should be acknowledged. First, several studies with small sample size included in our analysis might be underpowered to detect the relationship. Second, our results are based on the unadjusted parameters. A more accurate analysis should be performed, in which the outcomes should be adjusted by some related parameters, including age, menopause status, and other important lifestyle factors.

In summary, our meta-analysis might be the first meta-analysis to estimate the association of CYP $2 D 6 * 10$ polymorphism with the efficacy of tamoxifen treatment in female Asian breast cancer patients. Significant association of $* 10 / * 10(T T)$ genotype with poorer DFS and recurrence was found in the Asian population. In the future, large and well-designed studies are required to illustrate the interactions of CYP2D6 genetic variants, including *10 polymorphism and tamoxifen response in female breast cancer patients.

\section{Acknowledgment}

The studies included obtained informed consent from all individual participants included in these studies.

\section{Disclosure}

The authors report no conflicts of interest in this work.

\section{References}

1. Early Breast Cancer Trialists' Collaborative Group (EBCTCG), Davies C, Godwin J, et al. Relevance of breast cancer hormone receptors and other factors to the efficacy of adjuvant tamoxifen: patient-level meta-analysis of randomised trials. Lancet. 2011;378(9793):771-784.

2. Fabian C, Tilzer L, Sternson L. Comparative binding affinities of tamoxifen, 4-hydroxytamoxifen, and desmethyltamoxifen for estrogen receptors isolated from human breast carcinoma: correlation with blood levels in patients with metastatic breast cancer. Biopharm Drug Dispos. 1981;2(4):381-390.

3. Klein DJ, Thorn CF, Desta Z, Flockhart DA, Altman RB, Klein TE. PharmGKB summary: tamoxifen pathway, pharmacokinetics. Pharmacogenet Genomics. 2013;23(11):643-647.

4. Kiyotani K, Mushiroda T, Imamura CK, et al. Significant effect of polymorphisms in CYP2D6 and ABCC2 on clinical outcomes of adjuvant tamoxifen therapy for breast cancer patients. J Clin Oncol. 2010; 28(8):1287-1293.

5. Schroth W, Goetz MP, Hamann U, et al. Association between CYP2D6 polymorphisms and outcomes among women with early stage breast cancer treated with tamoxifen. JAMA. 2009;302(13):1429-1436.

6. Margolin S, Lindh JD, Thoren L, et al. CYP2D6 and adjuvant tamoxifen: possible differences of outcome in pre- and post-menopausal patients. Pharmacogenomics. 2013;14(6):613-622.

7. Teh LK, Mohamed NI, Salleh MZ, et al. The risk of recurrence in breast cancer patients treated with tamoxifen: polymorphisms of CYP2D6 and ABCB1. AAPS J. 2012;14(1):52-59.

8. Goetz MP, Suman VJ, Hoskin TL, et al. CYP2D6 metabolism and patient outcome in the Austrian Breast and Colorectal Cancer Study Group trial (ABCSG) 8. Clin Cancer Res. 2013;19(2):500-507.

9. Bonanni B, Macis D, Maisonneuve P, et al. Polymorphism in the CYP2D6 tamoxifen-metabolizing gene influences clinical effect but not hot flashes: data from the Italian Tamoxifen Trial. $J$ Clin Oncol. 2006;24(22):3708-3709. 
10. Goetz MP, Schaid DJ, Wickerham DL, et al. Evaluation of CYP2D6 and efficacy of tamoxifen and raloxifene in women treated for breast cancer chemoprevention: results from the NSABP P1 and P2 clinical trials. Clin Cancer Res. 2011;17(21):6944-6951.

11. Rae JM, Drury S, Hayes DF, et al. CYP2D6 and UGT2B7 genotype and risk of recurrence in tamoxifen-treated breast cancer patients. J Natl Cancer Inst. 2012;104(6):452-460.

12. Regan MM, Leyland-Jones B, Bouzyk M, et al; Breast International Group (BIG) 1-98 Collaborative Group. CYP2D6 genotype and tamoxifen response in postmenopausal women with endocrine-responsive breast cancer: the breast international group 1-98 trial. J Natl Cancer Inst. 2012;104(6):441-451.

13. Sestak I, Kealy R, Nikoloff M, et al. Relationships between CYP2D6 phenotype, breast cancer and hot flushes in women at high risk of breast cancer receiving prophylactic tamoxifen: results from the IBIS-I trial. Br J Cancer. 2012;107(2):230-233.

14. Kiyotani K, Mushiroda T, Zembutsu H, Nakamura Y. Important and critical scientific aspects in pharmacogenomics analysis: lessons from controversial results of tamoxifen and CYP2D6 studies. J Hum Genet. 2013;58(6):327-333.

15. Lien EA, Soiland H, Lundgren S, et al. Serum concentrations of tamoxifen and its metabolites increase with age during steady-state treatment. Breast Cancer Res Treat. 2013;141(2):243-248.

16. Zanger UM, Raimundo S, Eichelbaum M. Cytochrome P450 2D6: overview and update on pharmacology, genetics, biochemistry. Naunyn Schmiedebergs Arch Pharmacol. 2004;369(1):23-37.

17. Jung JA, Lim HS. Association between CYP2D6 genotypes and the clinical outcomes of adjuvant tamoxifen for breast cancer: a metaanalysis. Pharmacogenomics. 2014;15(1):49-60.

18. Sakuyama K, Sasaki T, Ujiie S, et al. Functional characterization of 17 CYP2D6 allelic variants (CYP2D6.2, 10, 14A-B, 18, 27, 36, 39, 47-51, 53-55, and 57). Drug Metab Dispos. 2008;36(12):2460-2467.

19. Gaedigk A, Gotschall RR, Forbes NS, Simon SD, Kearns GL, Leeder JS. Optimization of cytochrome P4502D6 (CYP2D6) phenotype assignment using a genotyping algorithm based on allele frequency data. Pharmacogenetics. 1999;9(6):669-682.

20. Johansson I, Oscarson M, Yue QY, Bertilsson L, Sjöqvist F, IngelmanSundberg M. Genetic analysis of the Chinese cytochrome P4502D locus: characterization of variant CYP2D6 genes present in subjects with diminished capacity for debrisoquine hydroxylation. Mol Pharmacol. 1994;46(3):452-459.

21. Higgins JP, Thompson SG, Deeks JJ, Altman DG. Measuring inconsistency in meta-analyzes. BMJ. 2003;327(7414):557-560.

22. Bohning D. Meta-analysis: a unifying meta-likelihood approach framing unobserved heterogeneity, study covariates, publication bias, and study quality. Methods Inf Med. 2005;44(1):127-135.

23. Tierney JF, Stewart LA, Ghersi D, Burdett S, Sydes MR. Practical methods for incorporating summary time-to-event data into metaanalysis. Trials. 2007;8:16.

24. Harbord RM, Egger M, Sterne JA. A modified test for small-study effects in meta-analyzes of controlled trials with binary endpoints. Stat Med. 2006;25(20):3443-3457.

25. Chamnanphon M, Pechatanan K, Sirachainan E, et al. Association of CYP2D6 and CYP2C19 polymorphisms and disease-free survival of Thai post-menopausal breast cancer patients who received adjuvant tamoxifen. Pharmgenomics Pers Med. 2013;6:37-48.

26. Kiyotani K, Mushiroda T, Sasa M, et al. Impact of CYP2D6*10 on recurrence-free survival in breast cancer patients receiving adjuvant tamoxifen therapy. Cancer Sci. 2008;99(5):995-999.

27. Lei L, Wang X, Wu XD, et al. Association of CYP2D6*10 (c.100C > T) polymorphisms with clinical outcome of breast cancer after tamoxifen adjuvant endocrine therapy in Chinese population. Am J Transl Res. 2016;8(8):3585-3592.

28. Sensorn I, Sukasem C, Sirachainan E, et al. ABCB1 and ABCC2 and the risk of distant metastasis in Thai breast cancer patients treated with tamoxifen. Onco Targets Ther. 2016;9:2121-2129.
29. Sirachainan E, Jaruhathai S, Trachu N, et al. CYP2D6 polymorphisms influence the efficacy of adjuvant tamoxifen in Thai breast cancer patients. Pharmgenomics Pers Med. 2012;5:149-153.

30. Sukasem C, Sirachainan E, Chamnanphon M, et al. Impact of CYP2D6 polymorphisms on tamoxifen responses of women with breast cancer: a microarray-based study in Thailand. Asian Pac J Cancer Prev. 2012; 13(9):4549-4553.

31. Tian C, Yang Y, Li H. Correlation between CYP2D6* 10 gene polymorphism and prognosis of breast cancer patients treated with tamoxifen. Chin J Breast Dis. 2013;7(6):406-411.

32. Wang YY, Cheng GH, Pu ZC, et al. Polymorphisms of CYP2D6*10 and CYP2C19*2 and its effect on breast cancer patients with tamoxifen treatment. Chin Clin Pharmacol Ther. 2015;20(5):552-556.

33. Wei Y, Xu Z. Association between CYP2D6*10 genotype and survival of breast cancer patients receiving tamoxifen treatment. Anhui Med Pharm J. 2014;18(5):951-954.

34. Xiao G, Li D, Liu LX. Clinical snalyisi of CYP2D6 gene polymorphism and Tamoxifen efficacy in post-menopause breast cancer patients. J Ningxia Med Univ. 2014;36(3):315-318.

35. Xu Y, Sun Y, Yao L, et al. Association between CYP2D6 *10 genotype and survival of breast cancer patients receiving tamoxifen treatment. Ann Oncol. 2008;19(8):1423-1429.

36. Yu Y, You W, Liang D, et al. Efects of CYP2 D6 gene polymorphism on prognosis of ER-positive breast cancer patients treated with tamoxifen therapy. Shandong Med. 2016;56(42):17-20.

37. Zeng Y, Huang K, Huang W. The effect analysis of CYP2D6 gene polymorphism in the toremifene and tamoxifen treatment in patient with breast cancer. Pak J Pharm Sci. 2017;30(3(Special)):1095-1098.

38. Zhang X, Pu Z, Ge J, Shen J, Yuan X, Xie H. Association of CYP2D6*10, OATP1B1 A388G, and OATP1B1 T521C polymorphisms and overall survival of breast cancer patients after tamoxifen therapy. Med Sci Monit. 2015;21:563-569.

39. Novillo A, Romero-Lorca A, Gaibar M, Rubio M, FernándezSantander A. Tamoxifen metabolism in breast cancer treatment: taking the focus off the CYP2D6 gene. Pharmacogenomics J. 2017;17(2): $109-111$.

40. Areepium N, Panomvana D, Rungwanonchai P, Sathaporn S, Voravud N. Effects of CYP2D6 and UGT2B7 polymorphisms on pharmacokinetics of tamoxifen in Thai breast cancer patients. Breast Cancer (Dove Med Press). 2013;5:73-78.

41. Del Re M, Rofi E, Citi V, et al. Should CYP2D6 be genotyped when treating with tamoxifen? Pharmacogenomics. 2017;18(8):755-756.

42. Province MA, Goetz MP, Brauch H, et al; International Tamoxifen Pharmacogenomics Consortium. CYP2D6 genotype and adjuvant tamoxifen: meta-analysis of heterogeneous study populations. Clin Pharmacol Ther. 2014;95(2):216-227.

43. Johansson H, Gandini S, Serrano D, et al. A pooled analysis of CYP2D6 genotype in breast cancer prevention trials of low-dose tamoxifen. Breast Cancer Res Treat. 2016;159(1):97-108.

44. Lim HS, Ju Lee H, Seok Lee K, Sook Lee E, Jang IJ, Ro J. Clinical implications of CYP2D6 genotypes predictive of tamoxifen pharmacokinetics in metastatic breast cancer. J Clin Oncol. 2007;25(25): 3837-3845.

45. Okishiro M, Taguchi T, Jin Kim S, Shimazu K, Tamaki Y, Noguchi S. Genetic polymorphisms of CYP2D6 10 and CYP2C192, 3 are not associated with prognosis, endometrial thickness, or bone mineral density in Japanese breast cancer patients treated with adjuvant tamoxifen. Cancer. 2009;115(5):952-961.

46. Abreu MH, Gomes M, Menezes F, et al. CYP2D6*4 polymorphism: a new marker of response to hormonotherapy in male breast cancer? Breast. 2015;24(4):481-486.

47. Dezentje VO, van Schaik RH, Vletter-Bogaartz JM, et al. CYP2D6 genotype in relation to tamoxifen efficacy in a Dutch cohort of the tamoxifen exemestane adjuvant multinational (TEAM) trial. Breast Cancer Res Treat. 2013;140(2):363-373. 
OncoTargets and Therapy

\section{Publish your work in this journal}

OncoTargets and Therapy is an international, peer-reviewed, open access journal focusing on the pathological basis of all cancers, potential targets for therapy and treatment protocols employed to improve the management of cancer patients. The journal also focuses on the impact of management programs and new therapeutic agents and protocols on

patient perspectives such as quality of life, adherence and satisfaction. The manuscript management system is completely online and includes a very quick and fair peer-review system, which is all easy to use. Visit http://www.dovepress.com/testimonials.php to read real quotes from published authors.

Submit your manuscript here: http://www.dovepress.com/oncotargets-and-therapy-journal 